\title{
circUBAP2 regulates osteosarcoma progression via the miR-204-3p/HMGA2 axis
}

\author{
WEIGUO MA, NING XUE, JUNHUA ZHANG, DAN WANG, XIAOBIN YAO, LIN LIN and QINGXIA XU \\ Department of Clinical Laboratory, Affiliated Cancer Hospital of Zhengzhou University, \\ Henan Cancer Hospital, Zhengzhou, Henan 450008, P.R. China
}

Received November 11, 2020; Accepted January 19, 2021

DOI: 10.3892/ijo.2021.5178

\begin{abstract}
Circular RNA (circRNA/circ)-ubiquitin associated protein 2 (UBAP2), a newly recognized circRNA, serves a functional role in several types of tumor, including ovarian cancer, colorectal cancer and osteosarcoma. However, the precise roles and molecular mechanism underlying circUBAP2 in osteosarcoma (OS) are not completely understood. In the present study, the expression levels of circUBAP2, microRNA (miR)-204-3p and (HMGA2) were evaluated via reverse transcription-quantitative PCR in OS tissues and cells. OS cell proliferation, migration, invasion and apoptosis were assessed by performing Cell Counting Kit-8, Transwell and flow cytometry assays, respectively. HMGA2 protein expression levels were determined via western blotting. Dual-luciferase reporter assays were performed to verify the interaction between circUBAP2 and miR-204-3p, and between miR-204-3p and HMGA2. An RNA immunoprecipitation (RIP) assay was conducted to confirm the interaction between circUBAP2 and miR-204-3p. The results demonstrated that circUBAP2 expression was significantly upregulated in OS tissues and cell lines compared with paracancerous tissues and hFOB1.19 cells, respectively. In addition, high circUBAP2 expression levels in patients with OS were associated with a lower survival rate compared with lower expression levels in patients with OS. The functional assays revealed that circUBAP2 knockdown significantly inhibited OS cell proliferation, migration and invasion, but increased OS cell apoptosis compared with the small interfering RNA-negative control (si-NC) group. The dual-luciferase reporter and RIP assay results confirmed that circUBAP2 bound to miR-204-3p. Moreover, miR-204-3p expression was significantly downregulated in OS tissues compared with paracancerous tissues, and miR-204-3p expression was
\end{abstract}

Correspondence to: Dr Qingxia Xu, Department of Clinical Laboratory, Affiliated Cancer Hospital of Zhengzhou University, Henan Cancer Hospital, 127 Dongming Road, Jinshui, Zhengzhou, Henan 450008, P.R. China

E-mail: hnxqx12@163.com

Key words: circular RNA-ubiquitin associated protein 2, high mobility group A2, migration, osteosarcoma, microRNA-204-3p negatively correlated with circUBAP2 expression in OS tissues. Collectively, the results demonstrated that miR-204-3p was associated with circUBAP2 knockdown-mediated inhibition of OS cell malignant behavior. Moreover, miR-204-3p was also identified as one of the direct targets of HMGA2. Collectively, the results indicated that compared with the si-NC group, circUBAP2 knockdown significantly inhibited OS cell malignant behavior by binding to miR-204-3p, which subsequently regulated HMGA2 expression. Therefore, the present study demonstrated that circUBAP2 expression was upregulated in OS, and circUBAP2 regulated OS cell malignant behavior via the miR-204-3p/HMGA2 axis.

\section{Introduction}

Osteosarcoma (OS) typically occurs in adolescents and children, and is characterized by rapid progression, a high degree of malignancy, poor prognosis, poor outcomes resulting from chemotherapy or radiotherapy and a tendency towards lung metastasis (1). With advancements in surgery, chemotherapy and radiotherapy, the long-term survival rate of patients with OS has greatly improved (2); however, the overall prognosis of patients with OS remains poor, especially for those with distant metastasis or recurrent cancer (3). Therefore, identifying the mechanisms underlying OS is important for the development of novel diagnostic and therapeutic strategies.

Circular RNAs (circRNAs/circs) are a class of double-stranded closed RNAs lacking a 3 ' cap structure and a 5' poly tail, which are stable entities that exist widely in almost all eukaryotes (4). The majority of circRNAs that have been discovered and identified are non-coding RNAs (ncRNAs) that do not encode proteins, but are able to regulate gene expression (5). Previous studies have demonstrated that circRNAs are associated with atherosclerosis, pulmonary fibrosis, tumors, heart failure, Alzheimer's disease and myocardial injury (6-11). Accumulating evidence has also revealed the differential expression of circRNAs, including circ-SFMBT2, circ_0068033, circ_0043265, circHIAT1 and circ-MYBL2, in several types of cancer (12-16).

circ-ubiquitin associated protein 2 (UBAP2), a newly identified circRNA, has been reported to serve as an oncogene in several types of cancer(17-22). For example, circUBAP2 inhibits esophageal squamous cell carcinoma cell malignant behavior by modulating the microRNA (miRNA/miR)-422a/RAB10, 
member RAS oncogene family axis, and in triple-negative breast cancer cells via the miR-661/metastasis associated 1 axis $(18,19)$. In addition, in ovarian cancer (OC), circUBAP2 promotes OC progression by binding to miR-144, thereby regulating chromodomain-helicase-DNA-binding protein 2 (19). In OS, circUBAP2 promoted OS progression by sponging miR-143 and regulating the miR-641/Yes1 associated transcriptional regulator axis $(21,22)$. However, the molecular mechanism underlying circUBAP2 in OS is not completely understood.

miRNAs, which are 20 nucleotides in length, inhibit the expression of their target genes by binding to the 3 ' non-coding region of target gene mRNAs (23). Previous studies have demonstrated that miRNAs, including miR-192, miR-183 and miR-375, fulfill crucial roles with respect to OS cell proliferation, migration, invasion and drug resistance (24-26). miR-204-3p inhibits bladder cancer cell proliferation and enhances glioma cell apoptosis $(27,28)$. However, the roles of miR-204-3p in OS are not completely understood.

High-mobility group protein A2 (HMGA2) is a member of the high-mobility group protein family, which is highly expressed in certain types of malignant tumor, including breast, non-small cell lung and pancreatic cancer, as well as retinoblastoma (29-32). Increasing evidence has demonstrated that HMGA2 is involved in malignant transformation and epithelial-mesenchymal transition (EMT) of cells (33-35). For example, Zha et al (33) reported that HMGA2 participated in EMT, activating the Wnt/ $\beta$-catenin signaling pathway in gastric cancer. In addition, Zhao et al (34) reported that in tongue squamous cell carcinoma, HMGA2 contributed to cancer progression and metastasis by enhancing the EMT signaling pathway. Hawsawi et al (35) demonstrated that HMGA2 promoted prostate tumor progression by inducing EMT via the MAPK signaling pathway. Moreover, HMGA2 was reported to be negatively regulated by miRNAs, including miR-590, miR-219-5p, miR-497 and miR-493 (36-39). However, whether HMGA2 is regulated by miR-204-3p in OS requires further investigation.

Recently, a number of studies investigating circRNAs have focused on elucidating their underlying mechanism, for example, determining which circRNAs serve as miRNA sponges to competitively bind to miRNAs, thereby inhibiting the degradation of corresponding mRNAs and regulating gene expression (40-42). For example, Sang et al (40) demonstrated that circRNA_0025202 served as a sponge for miR-182-5p and further regulated the expression and activity of forkhead box $\mathrm{O} 3$ in breast cancer. Song et al (41) reported that circRNA_101996 served as a sponge of miR-8075, which targeted TPX2 microtubule nucleation factor in cervical cancer cells to promote cervical cancer progression (41). In addition, Xu et al (42) reported that circTADA2A-E6 served as an miR-203a-3p sponge to target suppressor of cytokine signaling 3, suppressing breast cancer progression and metastasis. Therefore, the present study aimed to identify whether circUBAP2 regulated miR-204-3p to modulate HMGA2 in order to influence OS progression. In addition, the mechanism underlying circUBAP2/miR-204-3p/HMGA2 axis-mediated regulation of OS progression was investigated. The results of the present study may provide theoretical guidance for further understanding the molecular mechanism underlying OS.

\section{Materials and methods}

Human tissue collection. A total of 42 OS tissues and paired adjacent non-cancerous tissues (distance from tumor margin, $5 \mathrm{~cm}$ ) were collected from patients with OS (age range, 8-50 years; mean age, $22.83 \pm 12.78$ years; 20 male patients and 22 female patients) at the Affiliated Cancer Hospital of Zhengzhou University (Zhengzhou, China) between March 2017 and December 2019. Inclusion criteria were as follows: i) Tissues were obtained during surgery and diagnosed with OS by two pathologists; ii) patients had not received chemotherapy or radiotherapy; and iii) patients were willing to participate. The exclusion criteria were as follows: i) Patients with other diseases, including other tumors; ii) patients who received treatment prior to participation in the present study; and iii) patients who refused to participate in the study. All tissue samples were confirmed by a pathologist. Tissues were immediately placed in liquid nitrogen. The present study was approved by the ethics committee of Zhengzhou University (approval no. 201908). All the patients provided written informed consent prior to specimen acquisition.

Reverse transcription-quantitative PCR (RT-qPCR). Total RNA was extracted from cells and tissues using TRIzol ${ }^{\circledR}$ reagent (Invitrogen; Thermo Fisher Scientific, Inc.). RNA concentration and purity was detected using a NanoDrop spectrophotometer (Thermo Fisher Scientific, Inc.). Total RNA $(2 \mu \mathrm{g})$ was reverse-transcribed into cDNA using the SuperScript VILO cDNA Kit (Invitrogen; Thermo Fisher Scientific, Inc.), according to the manufacturer's protocol. Subsequently, qPCR was performed using the SYBR-Green PCR kit (Invitrogen; Thermo Fisher Scientific, Inc.). The sequences of the primers used for qPCR as listed in Table I. The following thermocycling conditions were used for qPCR analysis of circUBAP2 and HMGA2: $94^{\circ} \mathrm{C}$ for $30 \mathrm{sec} ; 40$ cycles of $94^{\circ} \mathrm{C}$ for $15 \mathrm{sec}$, $55^{\circ} \mathrm{C}$ for $30 \mathrm{sec}$ and $72^{\circ} \mathrm{C}$ for $90 \mathrm{sec}$. The following thermocycling conditions were used for qPCR analysis of miR-204-3p: $95^{\circ} \mathrm{C}$ for $15 \mathrm{sec}$, annealing at $60^{\circ} \mathrm{C}$ for $30 \mathrm{sec}$ and extension at $72^{\circ} \mathrm{C}$ for $30 \mathrm{sec}$. GAPDH was used as the internal standard for circUBAP2 and HMGA2, and U6 was used as the internal standard for miR-204-3p. Relative gene expression levels were quantified using the $2^{-\Delta \Delta \mathrm{Cq}}$ method (43).

Cell culture and cell transfection. Human OS cell lines (MG63, U-2OS, HOS and SaOS-2) were purchased from American Type Culture Collection and cultured in DMEM (Sangon Biotech Co., Ltd.) supplemented with 10\% FBS (Gibco; Thermo Fisher Scientific, Inc.) at $37^{\circ} \mathrm{C}$ with $5 \% \mathrm{CO}_{2}$. The normal osteoblast cell line (hFOB1.19) was also purchased from American Type Culture Collection and cultured in RPMI-1640 (HyClone; Cytiva) supplemented with 10\% FBS at $33.5^{\circ} \mathrm{C}$ with $5 \% \mathrm{CO}_{2}$.

Small interfering RNAs (siRNAs/si) targeting circUBAP2 (si-circUBAP2 $2^{1 \#}$ and si-circUBAP2 ${ }^{2 \#}$ ), negative control (NC) siRNA (si-NC), miR-204-3p mimic and mimic NC, HMGA2-overexpression plasmid (pcDNA3.1-HMGA2) and pcDNA3.1 were synthesized by Shanghai GenePharma Co., Ltd. HOS and SaOS-2 cells $\left(5 \times 10^{6}\right.$ cells $\left./ \mathrm{ml}\right)$ were seeded into 6-well plates. At $70 \%$ confluence, cells were transfected with si-circUBAP2 $2^{1 \#}$, si-circUBAP2 $2^{2 \#}$, si-NC, miR-204-3p 
Table I. Sequences of siRNAs, mimics and primers used for reverse transcription-quantitative PCR.

\begin{tabular}{ll}
\hline Gene & \multicolumn{1}{c}{ Sequence $\left(5^{\prime} \rightarrow 3^{\prime}\right)$} \\
\hline si-circUBAP2 $^{1 *}$ & GCAGACUAUUCAGAUUCUACA \\
si-circUBAP2 $^{2 *}$ & GACUAUUCAGAUUCUACAUCU \\
circUBAP si-NC $^{\prime}$ & AAACGGUACCAUAUGGUAAGC \\
si-HMGA2 & GGGCAAUCUUAUAUAUCUA \\
HMGA2 si-NC & CCCAGUUACGAATCGCUUCCA \\
miR-204-3p mimic & GCTGGGAAGGCAAAGGGACGT \\
mimic NC & UCACGCCACCUUACUGGCGCC \\
circUBAP2 primer & F: CCAGTTCTTAGCCAGTTGA \\
& R: TGTCTCCAGGTGTTGATTC \\
miR-204-3p primer & F: GTTTGCTGGGAAGGCAAAG \\
& R: TGTTTTGCTGGGAAGGCAAA \\
HMGA2 primer & F: GGACAAGCAAGTTGATGAAT \\
& R: TGAGTGAGTAGACGAGTGA \\
U6 primer & F: CTCGCTTCGGCAGCACATATACT \\
$\beta$-actin primer & R: ACGCTTCACGAATTTGCGTGTC \\
& R: CCTGTACGCCAACACAGTGC \\
& R: ATACTCCTGCTTGCTGATCC \\
\hline
\end{tabular}

si, small interfering RNA; NC, negative control; circ, circular RNA; UBAP2, ubiquitin associated protein 2; miR, microRNA; HMGA2, high mobility group A2; F, forward; R, reverse.

mimic or mimic NC using Lipofectamine ${ }^{\circledR} 3000$ reagent (Invitrogen; Thermo Fisher Scientific, Inc.) at $37^{\circ} \mathrm{C}$. The following treatment groups were established: i) circUBAP2 si-NC group, $60 \mathrm{nM}$ si-NC; ii) si-circUBAP2 $2^{1 *}$ group, $60 \mathrm{nM}$ si-circUBAP2 $2^{1 *}$; iii) si-circUBAP2 $2^{2 \#}$ group, $60 \mathrm{nM}$ si-circUBAP2 ${ }^{2 *}$; iv) si-circUBAP2 + miR-204-3p mimic group, $60 \mathrm{nM}$ si-circUBAP2 $2^{1 \#}+40 \mathrm{nM}$ miR-204-3p mimic; v) mimic NC group, $40 \mathrm{nM}$ miR-204-3p mimic $\mathrm{NC}$; vi) miR-204-3p mimic group, $40 \mathrm{nM}$ miR-204-3p mimic; vii) HMGA2 si-NC group, $35 \mathrm{nM}$ si-HMGA2 NC; viii) si-HMGA2 group, $35 \mathrm{nM}$ si-HMGA2; ix) miR-204-3p mimic + si-HMGA2 group, $40 \mathrm{nM}$ miR-204-3p mimic + $35 \mathrm{nM}$ si-HMGA2; x) pc-DNA3.1 group, $1 \mu \mathrm{g} / \mu \mathrm{l}$ pc-DNA3.1; xi) si-circUBAP2 + pc-DNA3.1 group, $60 \mathrm{nM}$ si-circUBAP2 $2^{1 \#}$ $+1 \mu \mathrm{g} / \mu 1 \mathrm{pcDNA} 3.1$; and xii) si circUBAP2 + pc-HMGA2 group, $60 \mathrm{nM}$ si-circUBAP2 ${ }^{1 /}+1 \mu \mathrm{g} / \mu 1 \mathrm{pcDNA3} .1-\mathrm{HMGA} 2 \mathrm{i}$ At $48 \mathrm{~h}$ post-transfection, transfection efficiencies were determined. The sequences of the siRNAs, miR-mimics and NCs are presented in Table I.

Flow cytometric analysis of apoptosis. Briefly, cells were digested with $0.25 \%$ trypsin and a cell suspension was prepared. Following centrifugation at $4^{\circ} \mathrm{C}$ at $1,200 \mathrm{x}$ g for $10 \mathrm{~min}$, the supernatant was removed, precooled PBS was added to cells and the cells were centrifuged again at $4^{\circ} \mathrm{C}$ at $1,200 \mathrm{x}$ g for $10 \mathrm{~min}$. The supernatant was removed and cells were washed three times with PBS (5 min per wash). Subsequently, cell apoptosis was assessed using the Annexin V-FITC kit
(BD Biosciences) according to the manufacturer's protocol. Briefly, cells $\left(1 \times 10^{5}\right.$ cells $\left./ 100 \mu \mathrm{l}\right)$ were transferred to a $5 \mathrm{ml}$ culture tube and treated with Binding Buffer containing $5 \mu \mathrm{l}$ FITC Annexin V and $5 \mu \mathrm{l} \mathrm{PI}$ for $15 \mathrm{~min}$ at $25^{\circ} \mathrm{C}$ in the dark. Subsequently, $400 \mu 11 \mathrm{X}$ binding buffer was added to each tube. Cell apoptosis (early and late apoptosis) was detected using a FACSCalibur flow cytometer (BD Biosciences) within $1 \mathrm{~h}$ and analyzed using CellQuest software (version no. 643274; BD Biosciences).

Cell Counting Kit (CCK)-8 assay. Cells were seeded $\left(2 \times 10^{4}\right.$ cells/well) into 96 -well plates and cultured at $37^{\circ} \mathrm{C}$ with $5 \% \mathrm{CO}_{2}$. Following incubation for $1,2,3$ or 4 days, $10 \mu \mathrm{l} \mathrm{CCK}-8$ reagent (Beyotime Institute of Biotechnology) was added into each well and incubated for $4 \mathrm{~h}$. Absorbance was measured at a wavelength of $450 \mathrm{~nm}$ using a microplate reader. Each reading was based on an average of at least 5 replicate wells.

Transwell assay. Transwell invasion assays were performed using a Transwell chamber ( $8-\mu \mathrm{m}$; Corning, Inc.) containing a Matrigel-coated membrane (BD Bioscience). Tranwell migration assays were performed using a Trasnwell chamber without the Matrigel-coated membrane. Briefly, cells were digested with trypsin and resuspended in serum-free medium. Subsequently, $200 \mu \mathrm{l}$ cell suspension $\left(2 \times 10^{4}\right.$ cells/l) was plated into the upper chamber of the Transwell chamber and $700 \mu \mathrm{l}$ complete medium was added to the lower chamber. Following incubation for $48 \mathrm{~h}$ at $37^{\circ} \mathrm{C}$, the chamber was extracted and washed with PBS. Non-invading/migratory cells were removed using a wet cotton swab. Invading/migratory cells were fixed with $4 \%$ paraformaldehyde for $20 \mathrm{~min}$ at room temperature, washed at least three times with PBS and stained with $1 \%$ crystal violet solution at room temperature for $5 \mathrm{~min}$. Stained cells were counted using a light IX51 inverted microscope (Olympus Corporation; magnification, x200), and an average was calculated from five randomly selected fields.

Western blotting. Following washing with PBS, total protein was extracted from cells using RIPA lysate (Thermo Fisher Scientific, Inc.). Protein concentration was determined using BCA reagent (Invitrogen; Thermo Fisher Scientific, Inc.). Proteins $(30 \mu \mathrm{g})$ were separated via $10 \%$ SDS-PAGE and transferred to a PVDF membrane. Following blocking with $5 \%$ non-fat milk for $2 \mathrm{~h}$ in TBST $(0.1 \%$ Tween-20; Sangon Biotech Co., Ltd.) at room temperature, the membranes were incubated at $4{ }^{\circ} \mathrm{C}$ overnight with primary antibodies (diluted in 0.1\% Tween-20) targeted against: HMGA2 (cat. no. ab207301; 1:1,000; Abcam) and $\beta$-actin (cat. no. ab8227; 1:5,000; Abcam). Subsequently, the membranes were incubated with a HRP-conjugated secondary antibody (cat. no. ab6721; 1:5,000; Abcam) at room temperature for $2 \mathrm{~h}$. Following washing three times with TBST, protein bands were visualized using an enhanced chemiluminescence kit (Pierce; Thermo Fisher Scientific, Inc.). Protein expression was quantified using ImageJ software (version 4.0; Bio-Rad Laboratories, Inc.) with $\beta$-actin as the loading control.

Bioinformatics analysis. The circular RNA interactome database-CircBank (www.circbank.cn/searchCirc.html) was used to predict the candidate downstream targets of circUBAP2. 
TargetScan (www.targetscan.org/vert_71/) was used to predict the potential targets of miR-204-3p.

RNA immunoprecipitation (RIP) assay. RIP was performed using a Magna RNA-binding protein immunoprecipitation kit (EMD Millipore). At $90 \%$ confluence, HOS and SaOS-2 cells were centrifuged at $4^{\circ} \mathrm{C}$ for $5 \mathrm{~min}$ at $1,000 \mathrm{x} \mathrm{g}$, washed with pre-cooled PBS and lysed with RIP lysis buffer. Subsequently, the lysates were incubated for $10 \mathrm{~min}$ at $4^{\circ} \mathrm{C}$ with human argonaute RISC catalytic component 2 (Ago2) antibody (cat. no. ab186733; 1:50; Abcam; 5 $\mu \mathrm{g}$ ) conjugated on magnetic beads, with IgG antibody (cat. no. ab172730; 1:100; Abcam; $5 \mu \mathrm{g}$ ) as the control group. Samples were treated with Proteinase K for $30 \mathrm{~min}$ at $55^{\circ} \mathrm{C}$ with gentle agitation. Immunoprecipitated RNA was isolated using TRIzol. Co-precipitated RNAs were purified, identified and analyzed via RT-qPCR according to the aforementioned protocol.

RNase R treatment. The stability of RNA was determined using RNase R. Total RNA $(10 \mu \mathrm{g})$ isolated from HOS and SaOS-2 using TRIzol was mixed with 40 units RNase R (Epicentre Technologies Pvt. Ltd.) for $20 \mathrm{~min}$ at $37^{\circ} \mathrm{C}$ to remove the linear RNA. Subsequently, RT-qPCR was performed according to the aforementioned protocol to determine the expression levels of circUBAP2 and UBAP2.

Luciferase reporter assay. HOS and SaOS-2 cells were seeded $\left(3 \times 10^{5}\right.$ cells/well) into 24 -well plates. The wild-type (WT) and mutant (Mut) binding sites of the 3'-untranslated regions (3'-UTRs) of circUBAP2 and HMGA2 for miR-204-3p were amplified using PCR. Briefly, total DNA was extracted from HOS and SAOS-2 cells using the Animal Tissues/Cells Genomic DNA Extraction Kit (Beijing Solarbio Science \& Technology Co., Ltd.). DNA was amplified using 2X Taq PCR MasterMix (Beijing Solarbio Science \& Technology Co., Ltd.) and the following primers: circUBAP2 forward, 5'-CTGCCGTCAACTCCTGTTCT-3' and reverse, 5'-GCTGGCTCAACTGGCTAAGA-3'; and HMGA2 forward, 5'-TGAGCCCAGGAGTTTGAGAC-3' and reverse, 5'-GAAGGTCCCCAGAGGAAAGT-3'. The following thermocycling conditions were used for PCR: $3 \mathrm{~min}$ at $94^{\circ} \mathrm{C}$; followed by 35 cycles of $94^{\circ} \mathrm{C}$ for $30 \mathrm{sec}, 58^{\circ} \mathrm{C}$ for $30 \mathrm{sec}$ and $72^{\circ} \mathrm{C}$ for $45 \mathrm{sec}$. The WT and Mut $3^{\prime}$-UTRs were introduced into the pmirGLO luciferase expression vector (Promega Corporation) to construct the following plasmids, circUBAP2-WT, circUBAP2-Mut, HMGA2 3'-UTR-WT and HMGA2-Mut. HOS and SaOS-2 cells were co-transfected with $2.5 \mu \mathrm{g}$ pmirGLO-circUBAP2-WT, pmirGLO-HMGA2-WT, pmirGLO-circUBAP2-Mut or pmirGLO-HMGA2-Mut and $50 \mathrm{nM}$ miR-204-3p mimic or mimic NC using Lipofectamine 3000 at $37^{\circ} \mathrm{C}$. At $48 \mathrm{~h}$ post-transfection, luciferase activities were measured using the dual-luciferase reporter assay system (Promega Corporation) according to the manufacturer's protocol. Firefly luciferase activities were normalized to Renilla luciferase activities.

Statistical analysis. Statistical analyses were performed using SPSS (version 24.0; IBM Corp.) and GraphPad Prism (version 5; GraphPad Software, Inc.) software. Data are presented as the mean $\pm \mathrm{SD}$. All experiments were repeated at least three times. Comparisons between two groups, other than comparisons between tumor and adjacent non-tumor samples, were analyzed using the unpaired Student's t-test. Comparisons among multiple groups were analyzed using one-way ANOVA followed by Tukey's post hoc test. A paired Student's t-test was used to compare circUBAP2, miR-204-3p and HMGA2 expression levels between tumor and adjacent non-tumor samples. The $\chi^{2}$ test was used to analyze the relationship between circUBAP 2 expression and clinical parameters. Survival curves were assessed by performing Kaplan-Meier analysis followed by log-rank tests. The correlation between circUBAP2 expression and miR-204-3p/HMGA2 expression was determined by performing Spearman's correlation analysis. Receiver operating characteristic (ROC) analysis was performed to assess the sensitivity and specificity of the measured markers. The correlation between circUBAP2 and miR-204-3p, or miR-204-3p and HMGA2 in tissue samples was evaluated using Pearson's correlation coefficient. $\mathrm{P}<0.05$ was considered to indicate a statistically significant difference.

\section{Results}

circUBAP2 is upregulated in $O S$ and is associated with poor prognosis. First, RT-qPCR was performed to detect circUBAP2 expression levels, which were significantly higher in OS tissues compared with paracancerous tissues (Fig. 1A). Kaplan-Meier survival curve analysis demonstrated that patients with OS with high expression levels of circUBAP2 displayed lower survival rates compared with patients with OS with low expression levels of circUBAP2 (Fig. 1B). In addition, circUBAP2 expression was significantly associated with TNM stage (44) and distant metastasis (Table II). To further analyze whether circUBAP2 served as a tumor marker in OS, ROC curve analyses were performed. The results demonstrated that circUBAP2 effectively distinguished OS tissues from paired non-cancerous tissues (area under the curve $=0.7664$ and cutoff value $=0.524$; Fig. 1C). Subsequently, RT-qPCR was performed to evaluate circUBAP2 expression in OS cells. The results indicated that circUBAP2 expression levels were significantly increased in OS cell lines compared with human hFOB1.19 osteoblasts, particularly in HOS and SaOS-2 cells, which were selected for subsequent experiments (Fig. 1D). Following treatment with RNase, circUBAP2 expression was not significantly altered, whereas UBAP2 expression was significantly decreased compared with the Mock group, which confirmed the circRNA characteristics of circUBAP2 (Fig. 1E and F). Collectively, the results demonstrated that circUBAP2 was highly expressed in OS.

circUBAP2 knockdown inhibits OS cell proliferation, migration and invasion, and promotes $O S$ cell apoptosis. siRNAs (si-circUBAP2 $2^{1 \#}$ and si-circUBAP2 ${ }^{2 \#}$ ) were transfected into OS cells to assess the function of circUBAP2 in OS. At 48 h post-transfection, the RT-qPCR results demonstrated that compared with the si-NC group, circUBAP2 expression was significantly decreased in OS cells transfected with si-circUBAP2, indicating successful transfection (Fig. 2A). si-circUBAP $2^{1 \#}$ displayed higher transfection efficiency compared with si-circUBAP $2^{2 \#}$, thus $s i-c i r c U B A P 2^{1 \#}$ 

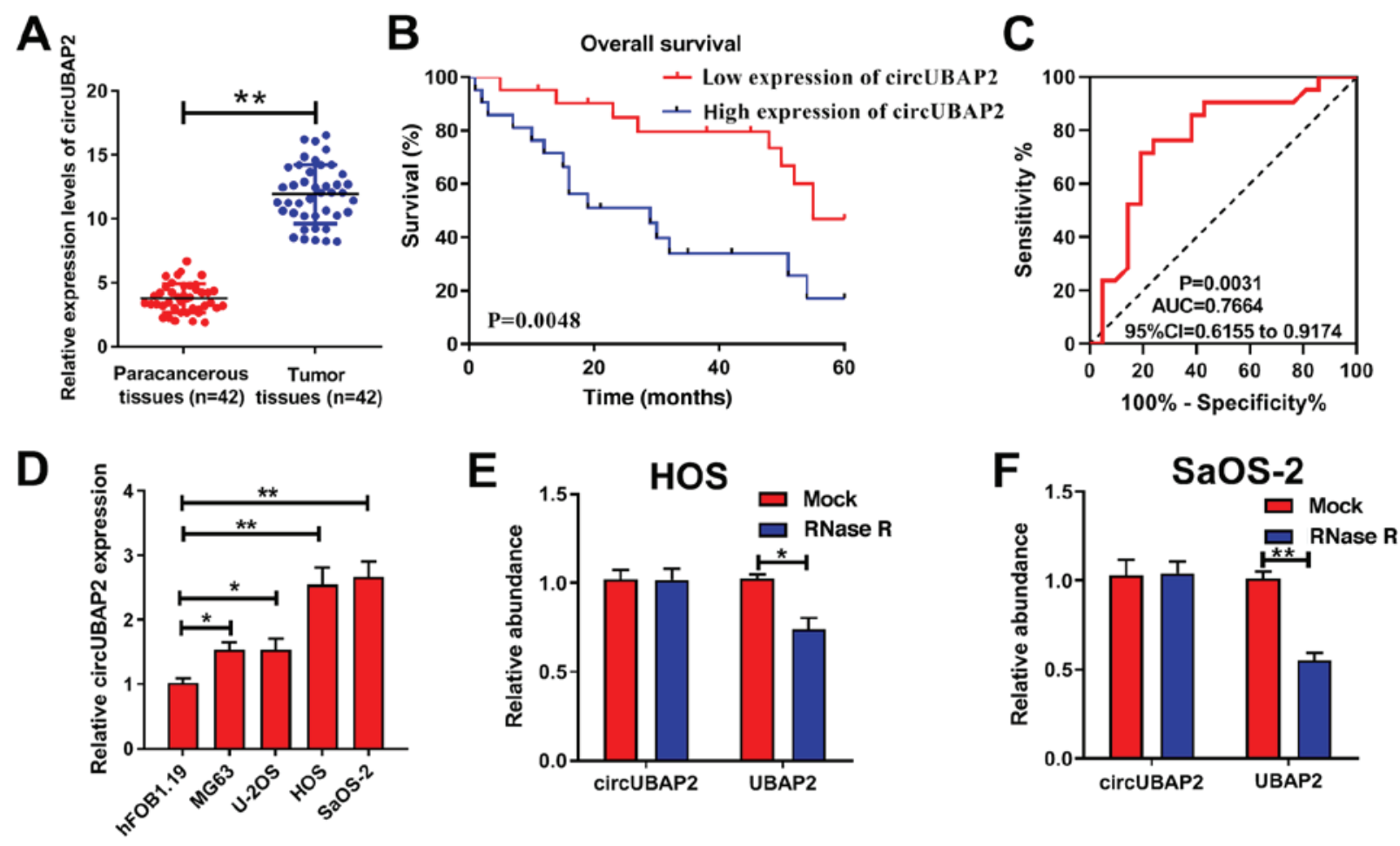

Figure 1. circUBAP2 expression is upregulated in OS and associated with poor prognosis. (A) circUBAP2 expression in OS tissues as determined via RT-qPCR. (B) Kaplan-Meier analysis demonstrated an association between circUBAP2 expression and overall survival in patients with OS. (C) Receiver operating characteristic curves indicated that circUBAP2 effectively distinguished OS tissues from paired non-cancerous tissues (AUC $=0.7664$; cut-off value $=0.524$ ). (D) circUBAP2 expression in OS cells as determined via RT-qPCR. RNase treatment in (E) HOS and (F) SaOS-2 cells significantly decreased UBAP2 expression, but did not significantly alter circUBAP2 expression. ${ }^{~} \mathrm{P}<0.05$ and ${ }^{* *} \mathrm{P}<0.01$. circ, circular RNA; UBAP2, ubiquitin associated protein 2 ; OS, osteosarcoma; RT-qPCR, reverse transcription-quantitative PCR; AUC, area under the curve; CI, confidence interval.

(si-circUBAP2) was used for subsequent experiments. The functional assays revealed that circUBAP2 knockdown significantly inhibited OS cell proliferation, migration and invasion, and increased OS cell apoptosis compared with the si-NC group (Fig. 2B-F), which demonstrated that circUBAP2 might serve as a tumor promoter in OS.

circUBAP2 sponges $m i R-204-3 p$, and $m i R-204-3 p$ is downregulated in OS. By analyzing the circular RNA interactome database, miR-204-3p was identified as a potential target of circUBAP2 (Fig. 3A). Subsequently, the dual-luciferase reporter assays results suggested that circUBAP2 could bind directly to miR-204-3p in OS cells (Fig. 3B and C). In addition, anti-Ago2 antibody was employed in an RIP assay to capture Ago2 protein and the binding RNAs. The results demonstrated that circUBAP2 could bind to miR-204-3p (Fig. 3D and E). The expression levels of circUBAP2 and miR-204-3p were subsequently detected via RT-qPCR. Compared with the si-NC group, miR-204-3p expression was significantly increased following circUBAP2 knockdown (Fig. 3F). In OS tissues, miR-204-3p expression was significantly downregulated compared with paracancerous tissues (Fig. 3G). Moreover, miR-204-3p expression was negatively correlated with circUBAP2 expression (Fig. 3H). Further analysis revealed that patients with OS with low expression levels of miR-204-3p displayed a lower overall survival rate compared with patients with OS with high expression levels of miR-204-3p (Fig. 3I). Moreover, miR-204-3p was expressed at significantly lower levels in OS cells compared with hFOB1.19 cells (Fig. 3J). Collectively, the results demonstrated that circUBAP2 sponged miR-204-3p, and miR-204-3p expression was downregulated in OS.

circ UBAP2 promotes $O S$ cell proliferation, migration and invasion, and inhibits $O S$ cell apoptosis by sponging $m i R-204-3 p$. The transfection efficiency of miR-204-3p is presented in Fig. 4A. Subsequently, HOS and SaOS-2 cells were co-transfected with miR-204-3p mimic and si-circUBAP2 $2^{1 \#}$ (si-circUBAP2). circUBAP2 knockdown significantly reduced OS cell proliferation, migration and invasion, and promoted OS cell apoptosis compared with the si-NC group. Following co-transfection with miR-204-3p mimic, circUBAP2 knockdown-mediated effects on proliferation, migration and invasion were significantly increased (Fig. 4B-E), whereas circUBAP2-knockdown-induced cell apoptosis was significantly inhibited, which suggested that miR-204-3p was associated with circUBAP2-mediated promotion of OS cell malignant behavior.

HMGA2 is a target of miR-204-3p. miRNAs are able to mediate downregulation of the expression of their target genes by targeting the mRNA 3'-UTR downstream of the target gene (45). TargetScan was used to identify HMGA2 as a potential target gene of miR-204-3p (Fig. 5A). To further confirm regulation of the HMGA2 gene by miR-204-3p, dual-luciferase reporter assays were performed. The results obtained verified the interaction between miR-204-3p and HMGA2 in HOS and SaOS-2 cells (Fig. 5B and C). In addition, the western blotting 


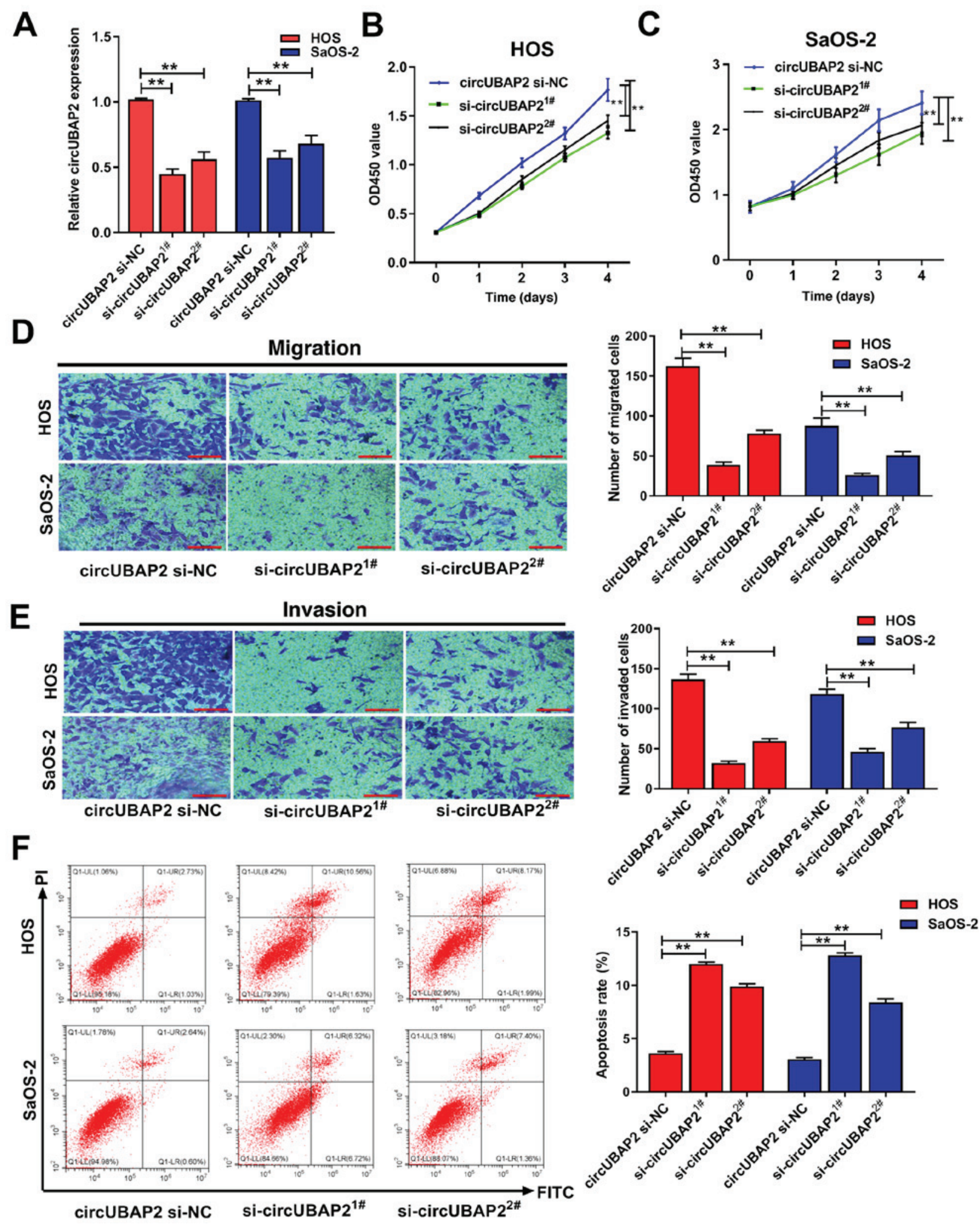

Figure 2. circUBAP2 knockdown inhibits OS cell proliferation, migration and invasion, and promotes OS cell apoptosis. (A) Transfection efficiency of si-circUBAP2 ${ }^{1 \#}$ and si-circUBAP2 ${ }^{2 *}$. Effect of circUBAP2 knockdown on (B) HOS and (C) SaOS-2 cell proliferation as determined by performing Cell Counting Kit-8 assays. Effect of circUBAP2 knockdown on HOS and SaOS-2 cell (D) migration and (E) invasion as determined by performing Transwell assays (scale bar, $500 \mu \mathrm{m}$ ). (F) Effect of circUBAP2 knockdown on HOS and SaOS-2 cell apoptosis as determined via flow cytometry. ${ }^{* *} \mathrm{P}<0.01$. circ, circular RNA; UBAP2, ubiquitin associated protein 2; OS, osteosarcoma; si, small interfering RNA; NC, negative control; OD, optical density; d, days.

results demonstrated that miR-204-3p overexpression significantly decreased the expression levels of HMGA2 in HOS and SaOS-2 cells compared with mimic NC (Fig. 5D). In OS tissues, HMGA2 expression levels were significantly higher compared with paracancerous tissues (Fig. 5E). Moreover, Pearson's correlation analysis demonstrated that HMGA2 expression levels were negatively correlated with miR-204-3p expression levels (Fig. 5F). 
A
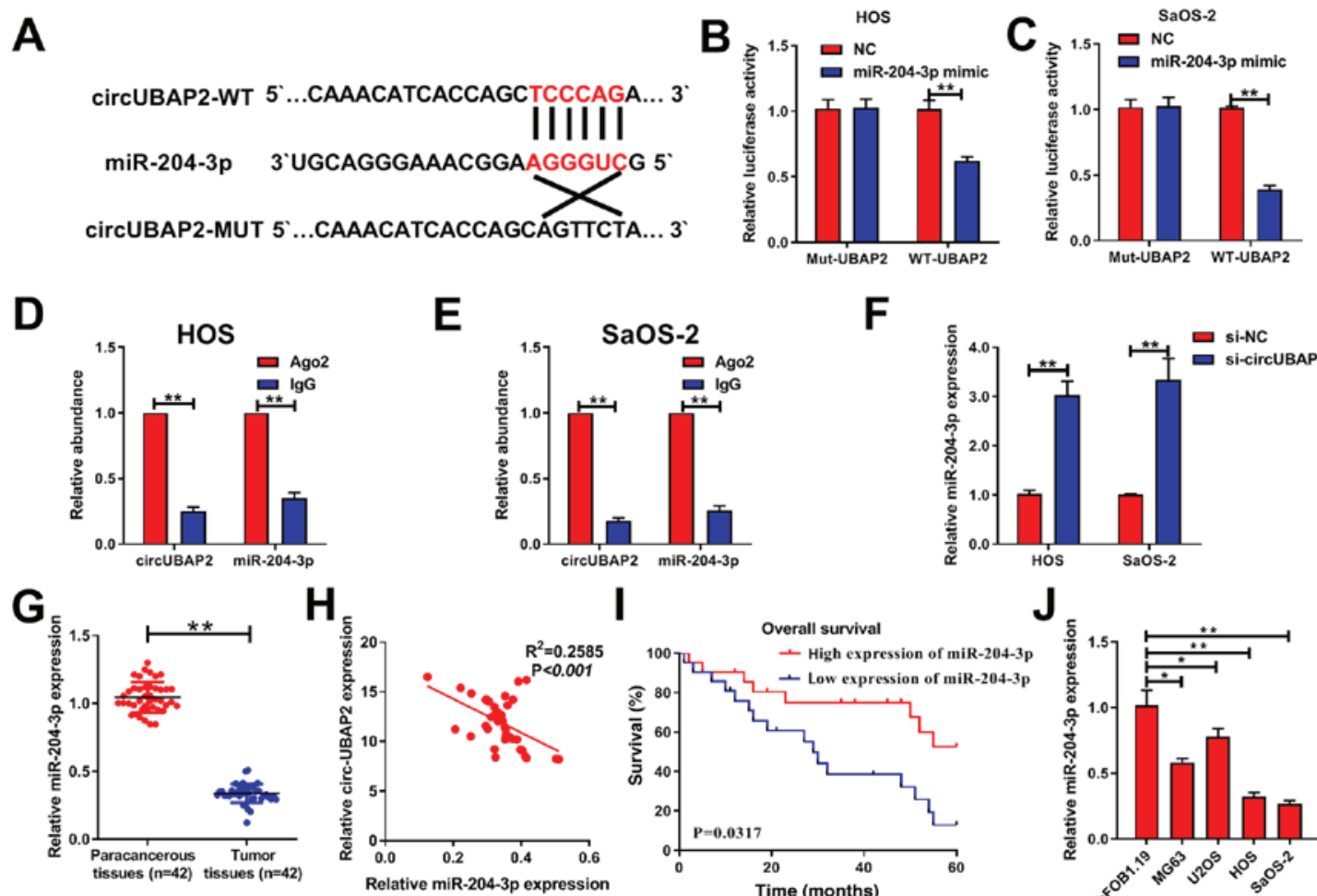

E

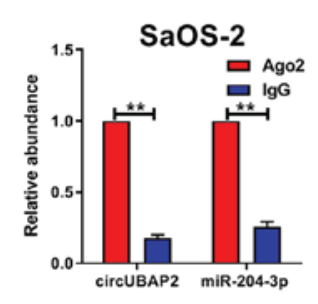

$\mathbf{F}$
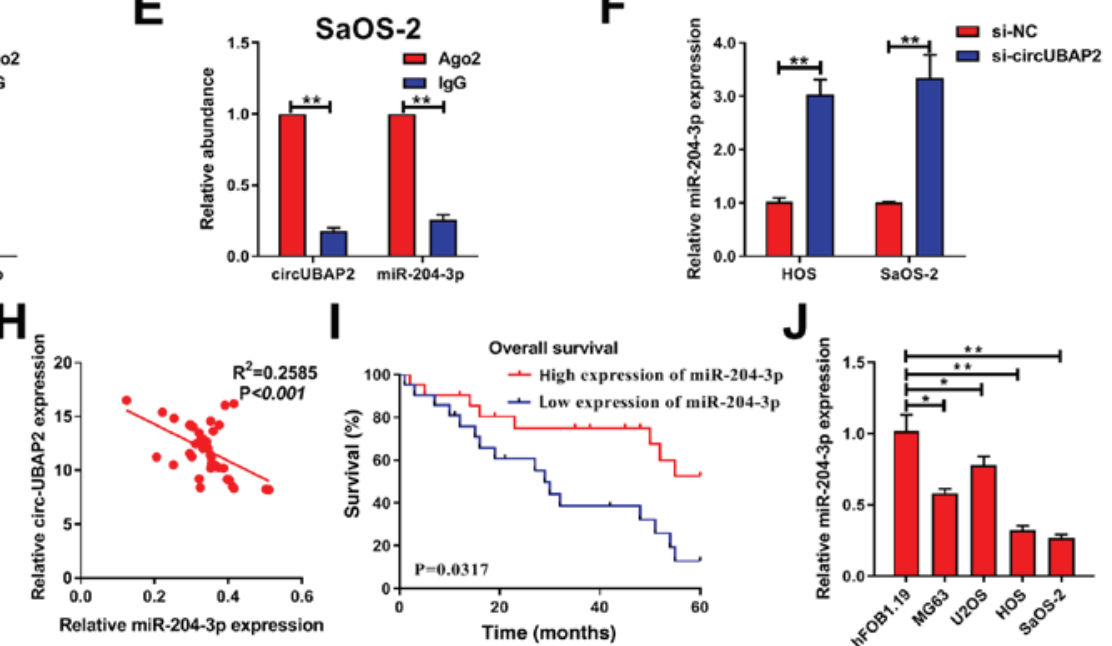

Figure 3. circUBAP2 sponge miR-204-3p and miR-204-3p is downregulated in OS. (A) Bioinformatics analysis predicted binding sites between miR-204-3p and circUBAP2. Luciferase reporter assays were performed to verify the interaction between miR-204-3p and circUBAP2 in (B) HOS and (C) SaOS-2 cells RNA immunoprecipitation assays were performed to further verify that circUBAP2 bound to miR-204-3p in (D) HOS and (E) SaOS-2 cells. (F) Effect of circUBAP2 knockdown on miR-204-3p expression in HOS and SaOS-2 cells as determined via RT-qPCR. (G) miR-204-3p expression in OS tissues as determined via RT-qPCR. (H) miR-204-3p expression was negatively correlated with circUBAP2 expression in OS tissues. (I) Kaplan-Meier analysis demonstrated an association between miR-204-3p expression and overall survival in patients with OS. (J) miR-204-3p expression in OS cells as determined via RT-qPCR. " $\mathrm{P}<0.05$ and ${ }^{* *} \mathrm{P}<0.01$. circ, circular RNA; UBAP2, ubiquitin associated protein 2; miR, microRNA; OS, osteosarcoma; RT-qPCR, reverse transcription-quantitative PCR; NC, negative control; WT, wild-type; Mut, mutant; Ago2, argonaute RISC catalytic component 2; si, small interfering RNA.

Table II. Association between circUBAP2 expression and clinical parameters of patients with osteosarcoma.

\begin{tabular}{|c|c|c|c|c|}
\hline \multirow[b]{2}{*}{ Clinical parameter } & \multirow[b]{2}{*}{$\mathrm{n}$} & \multicolumn{2}{|c|}{ circUBAP2 expression } & \multirow[b]{2}{*}{ P-value } \\
\hline & & Low $(n=21)$ & $\operatorname{High}(\mathrm{n}=21)$ & \\
\hline Sex & & & & 0.758 \\
\hline Male & 22 & 10 & 12 & \\
\hline Female & 20 & 11 & 9 & \\
\hline Age (years) & & & & 1.00 \\
\hline$<18$ & 25 & 13 & 12 & \\
\hline$\geq 18$ & 17 & 8 & 9 & \\
\hline Location & & & & 0.085 \\
\hline Femur & 30 & 12 & 18 & \\
\hline Other & 12 & 9 & 3 & \\
\hline Tumor size (cm) & & & & 0.111 \\
\hline$<8$ & 26 & 16 & 10 & \\
\hline$\geq 8$ & 16 & 5 & 11 & \\
\hline TNM stage & & & & $0.012^{\mathrm{a}}$ \\
\hline I-II & 23 & 16 & 7 & \\
\hline III & 19 & 5 & 14 & \\
\hline Distant metastasis & & & & $0.029^{\mathrm{a}}$ \\
\hline No & 20 & 14 & 6 & \\
\hline Yes & 22 & 7 & 15 & \\
\hline
\end{tabular}

${ }^{\mathrm{a}} \mathrm{P}<0.05$. circ, circular RNA; UBAP2, ubiquitin associated protein 2.
miR-204-3p inhibits OS cell proliferation, migration and invasion, and promotes $O S$ cell apoptosis by regulating HMGA2. To investigate whether HMGA2 was involved in miR-204-3p-induced OS cell malignant behavior, HOS and SaOS-2 cells were transfected with si-HMGA2 or si-NC. The transfection efficiency of si-HMGA2 is presented in Fig. 6A. Subsequently, HOS and SaOS-2 cells were co-transfected with miR-204-3p mimic and si-HMGA2. miR-204-3p overexpression significantly decreased OS cell proliferation, migration and invasion, and promoted OS cell apoptosis compared with mimic NC (Fig. 6B-E). miR-204-3p overexpression-mediated effects were significantly enhanced by HMGA2 knockdown. Collectively, the results demonstrated that HMGA2 was associated with miR-204-3p-mediated inhibition of OS cell malignant behavior.

circUBAP2 promotes OS cell proliferation, migration and invasion, and inhibits $O S$ cell apoptosis by regulating HMGA2. The aforementioned results demonstrated that circUBAP 2 promoted OS cell malignant behavior, and HMGA2 was a target of miR-204-3p, Therefore, whether circUBAP2 affected OS cell malignant behavior by regulating HMGA2 was examined. Firstly, pcDNA3.1-HMGA2 and pcDNA3.1 were transfected into cells (Fig. 7A). Subsequently, cells were co-transfected with pcDNA3.1-HMGA2 and si-circUBAP2. The results revealed that circUBAP2 knockdown significantly decreased OS cell 
A
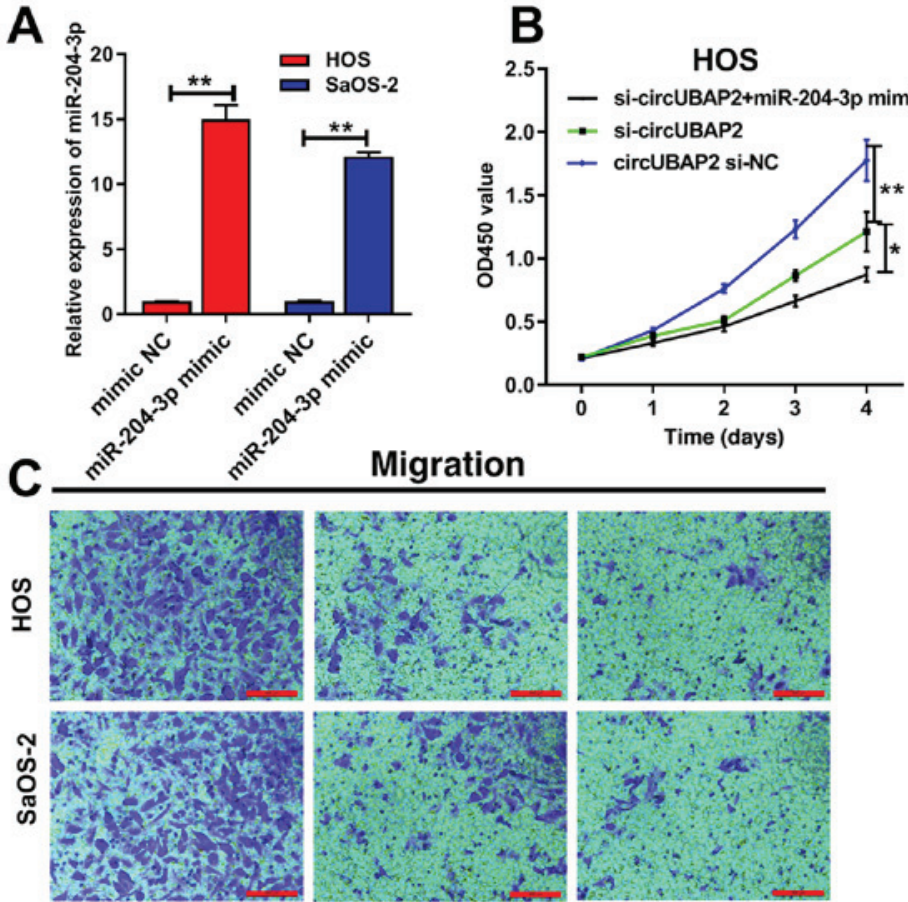

circUBAP2 si-NC

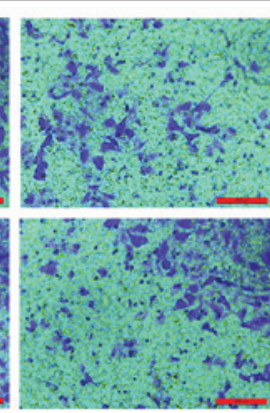

si-circUBAP2

Invasion

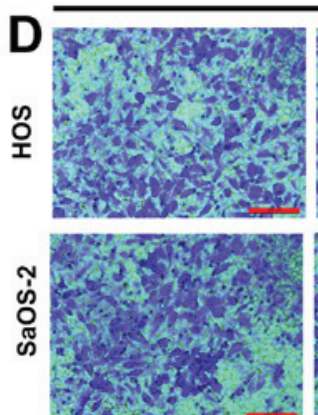

$E_{\bar{a}}$
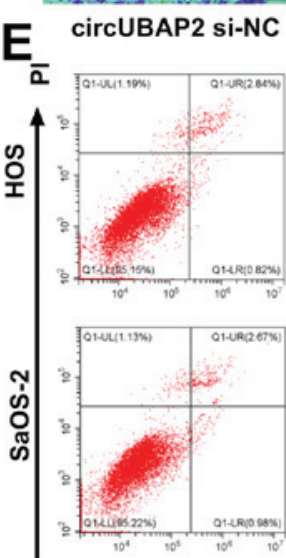

circUBAP2 si-NC

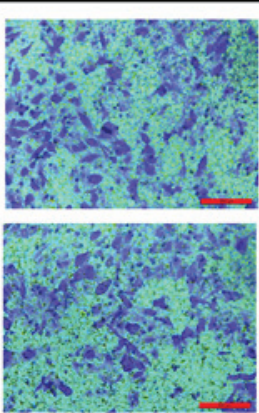

si-circUBAP2
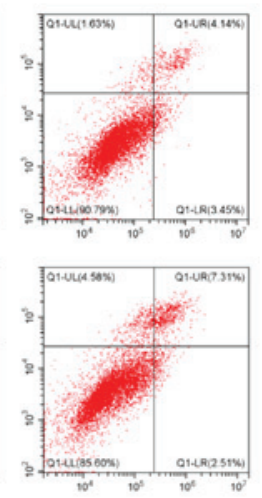

si-circUBAP2

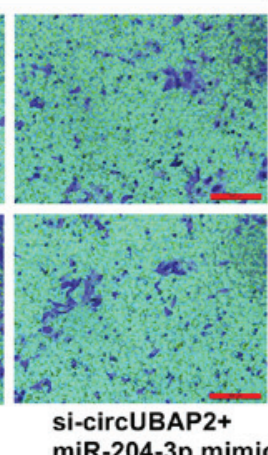

miR-204-3p mimic

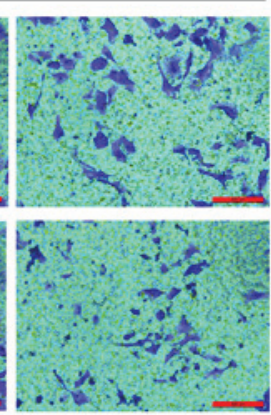

si-circUBAP2+ miR-204-3p mimic
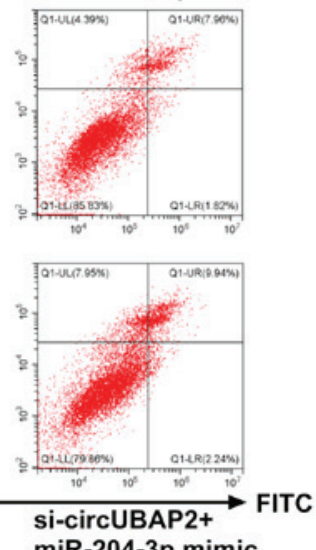
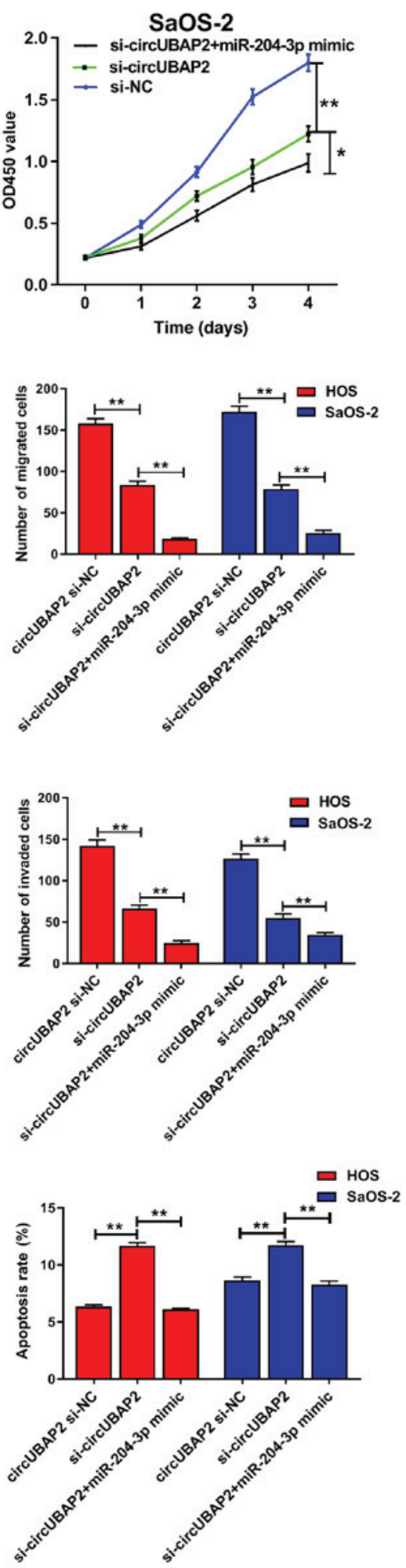

Figure 4. circUBAP2 promotes OS cell proliferation, migration and invasion, and inhibits OS cell apoptosis by sponging miR-204-3p. (A) Transfection efficiency of miR-203-3p mimic. (B) Cell Counting Kit-8 assays were performed to examine the effect of circUBAP2 and miR-204-3p on HOS and SaOS-2 cell proliferation. (C) Transwell assays were performed to assess the effect of circUBAP2 and miR-204-3p on HOS and SaOS-2 cell (C) migration and (D) invasion (scale bar, $500 \mu \mathrm{m}$ ). (E) Flow cytometry was performed to assess the effect of circUBAP2 and miR-204-3p on HOS and SaOS-2 cell apoptosis. " $\mathrm{P}<0.05$ and ${ }^{* *} \mathrm{P}<0.01$. circ, circular RNA; UBAP2, ubiquitin associated protein 2; OS, osteosarcoma; miR, microRNA; NC, negative control; si, small interfering RNA; OD, optical density.

proliferation, migration and invasion, and promoted OS cell apoptosis compared with the si-NC group. However, circUBAP2 knockdown-mediated effects were significantly reversed by HMGA2 overexpression, which further indicated that circUBAP2 affected OS cell biological behavior by regulating HMGA2 (Fig. 7B-E). 
A
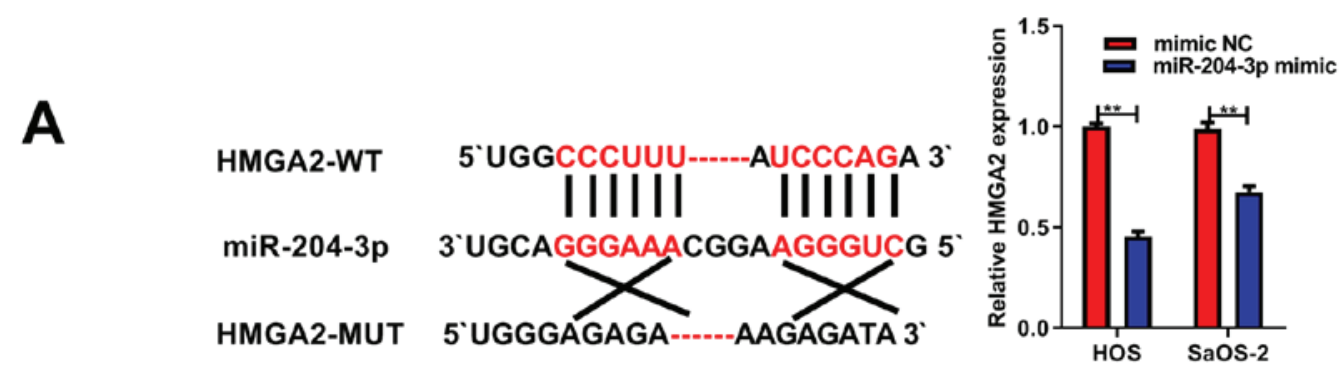

B

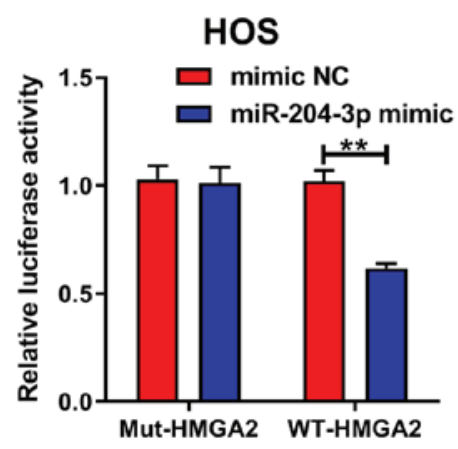

C

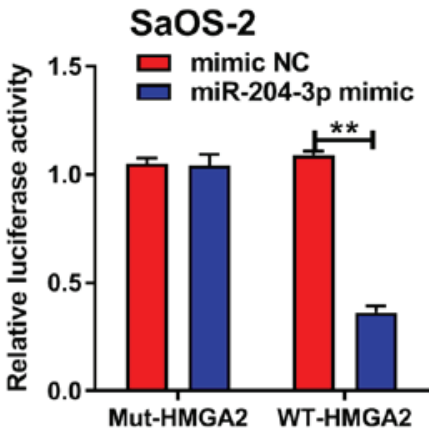

D
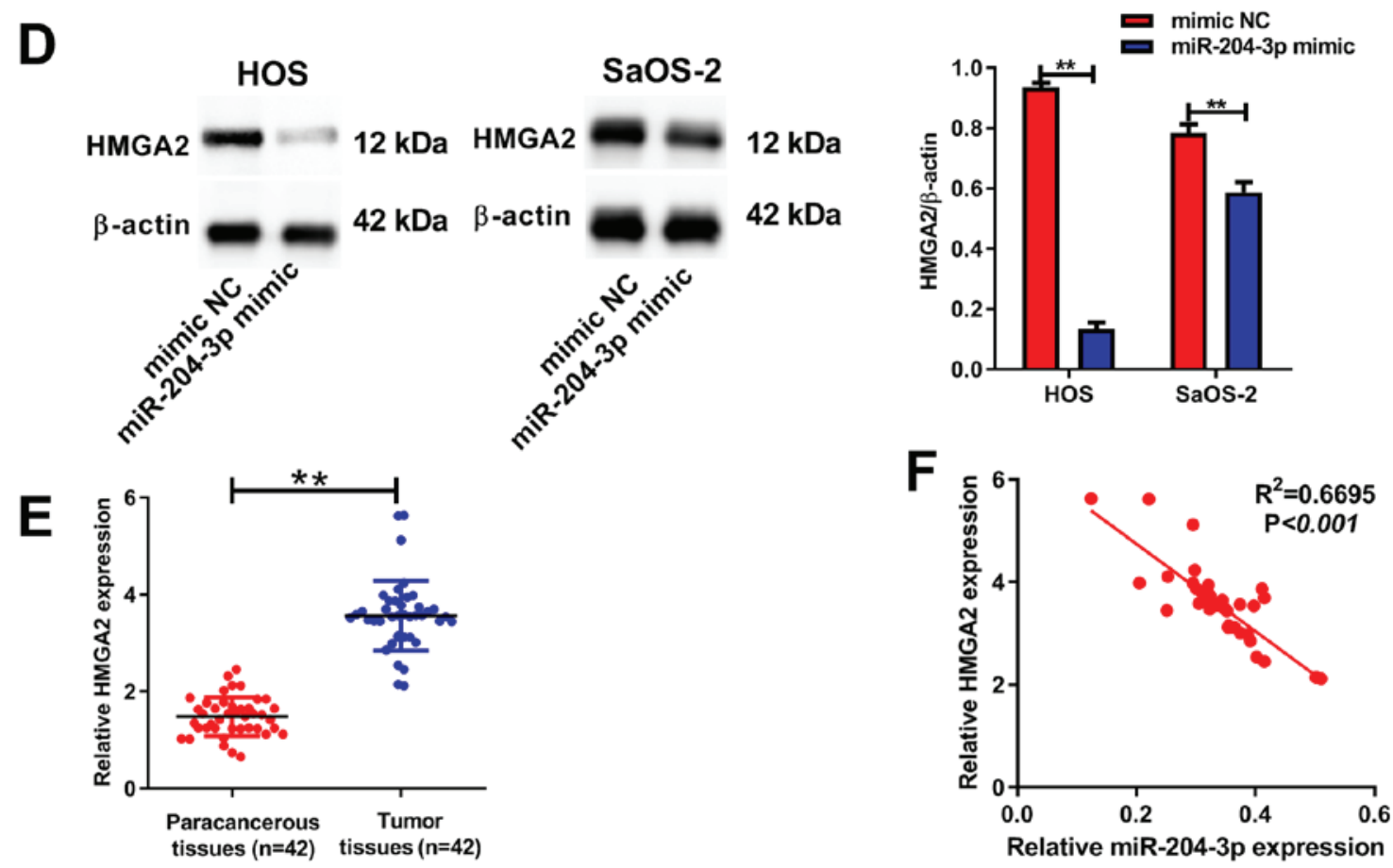

Figure 5. HMGA2 is a target of miR-204-3p. (A) Bioinformatics analysis predicted binding sites between miR-204-3p and HMGA2, and RT-qPCR was performed to assess the effect of miR-204-3p overexpression on HMGA2 expression. Luciferase assays were performed to verify the interaction between miR-204-3p and HMGA2 in (B) HOS and (C) SaOS-2 cells. (D) HMGA2 protein expression levels were determined via western blotting. (E) HMGA2 expression in OS tissues as determined via RT-qPCR. (F) HMGA2 expression was negatively correlated with miR-204-3p expression in OS tissues. ${ }^{* *} \mathrm{P}<0.01$. HMGA2, high mobility group A2; miR, microRNA; RT-qPCR, reverse transcription-quantitative PCR; OS, osteosarcoma; WT, wild-type; Mut, mutant; NC, negative control.

\section{Discussion}

Increasing evidence has demonstrated that ncRNAs are involved in the development of different types of cancer, including nasopharyngeal carcinoma, breast cancer, osteosarcoma and esophageal squamous cell carcinoma (46-50). circRNAs, which are a group of ncRNAs, are also associated with various diseases (51-55). Hao et al (56) reported that circ_0016760 promoted lung cancer cell proliferation by regulating the miR-577/zinc finger and BTB domain containing 7A axis. Wang et al (57) demonstrated that the circRNA mitochondrial fission and apoptosis-related could regulate expression of mitochondrial fission process 1 via miR-652-3p, thereby participating in the process of mitochondrial division. circUBAP2 has been reported to serve roles in several types of tumor, including clear cell renal cell carcinoma, esophageal squamous cell carcinoma, triple-negative breast cancer, ovarian cancer and osteosarcoma (17-21); however, the roles of circUBAP2 in OS are not completely understood.

In the present study, circUBAP2 expression was significantly upregulated in OS tissues and cell lines compared with paracancerous tissues and hFOB1.19 cells, respectively. 
A

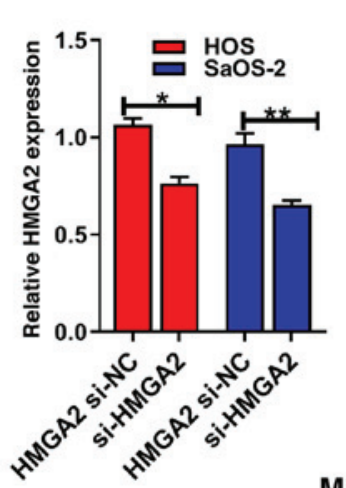

C

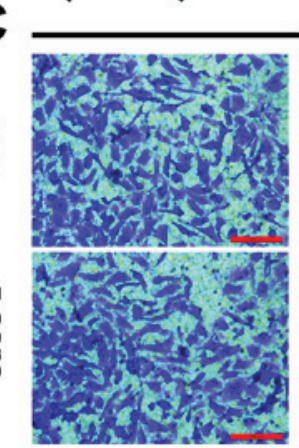

mimic NC

D

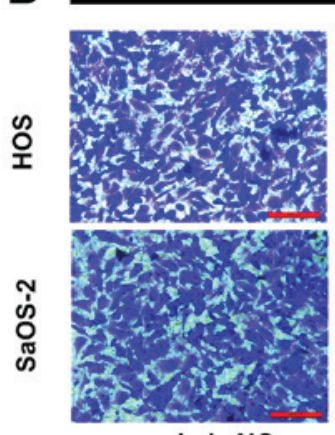

mimic NC

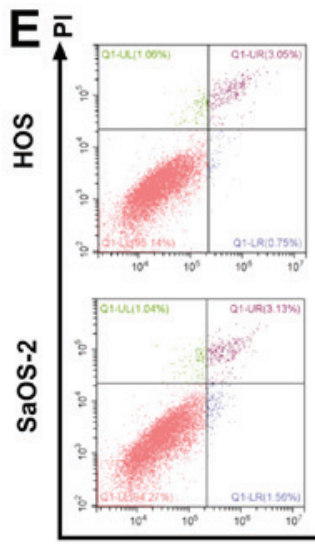

mimic NC
B

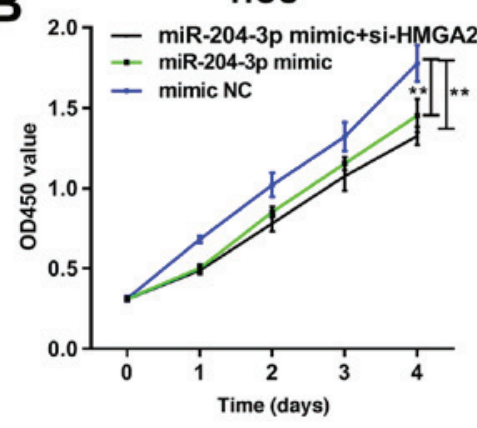

SaOS-2

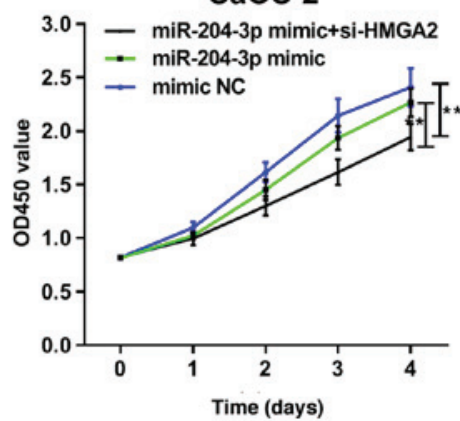

Migration

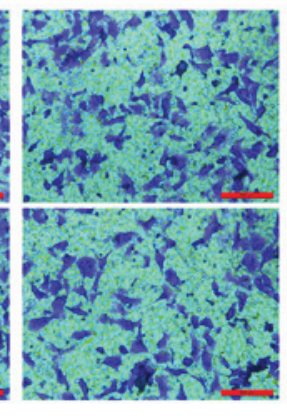

miR-204-3p mimic

Invasion

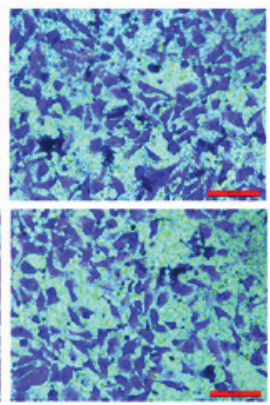

miR-204-3p mimic

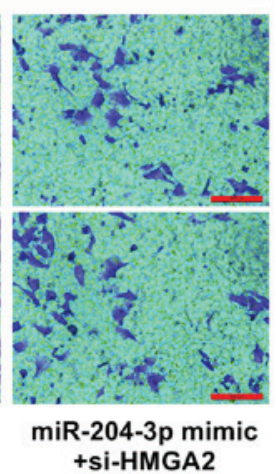

+si-HMGA2

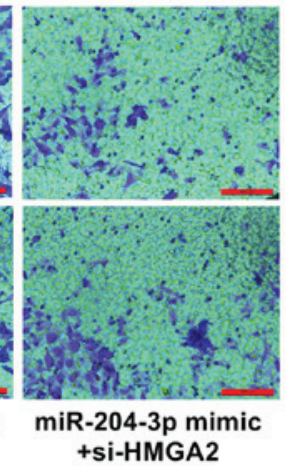

+si-HMGA2
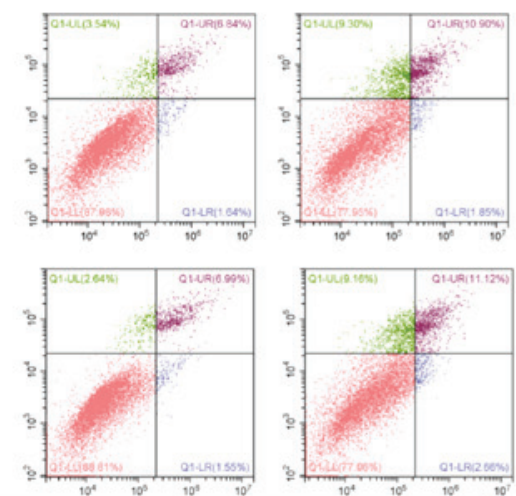

miR-204-3p mimic
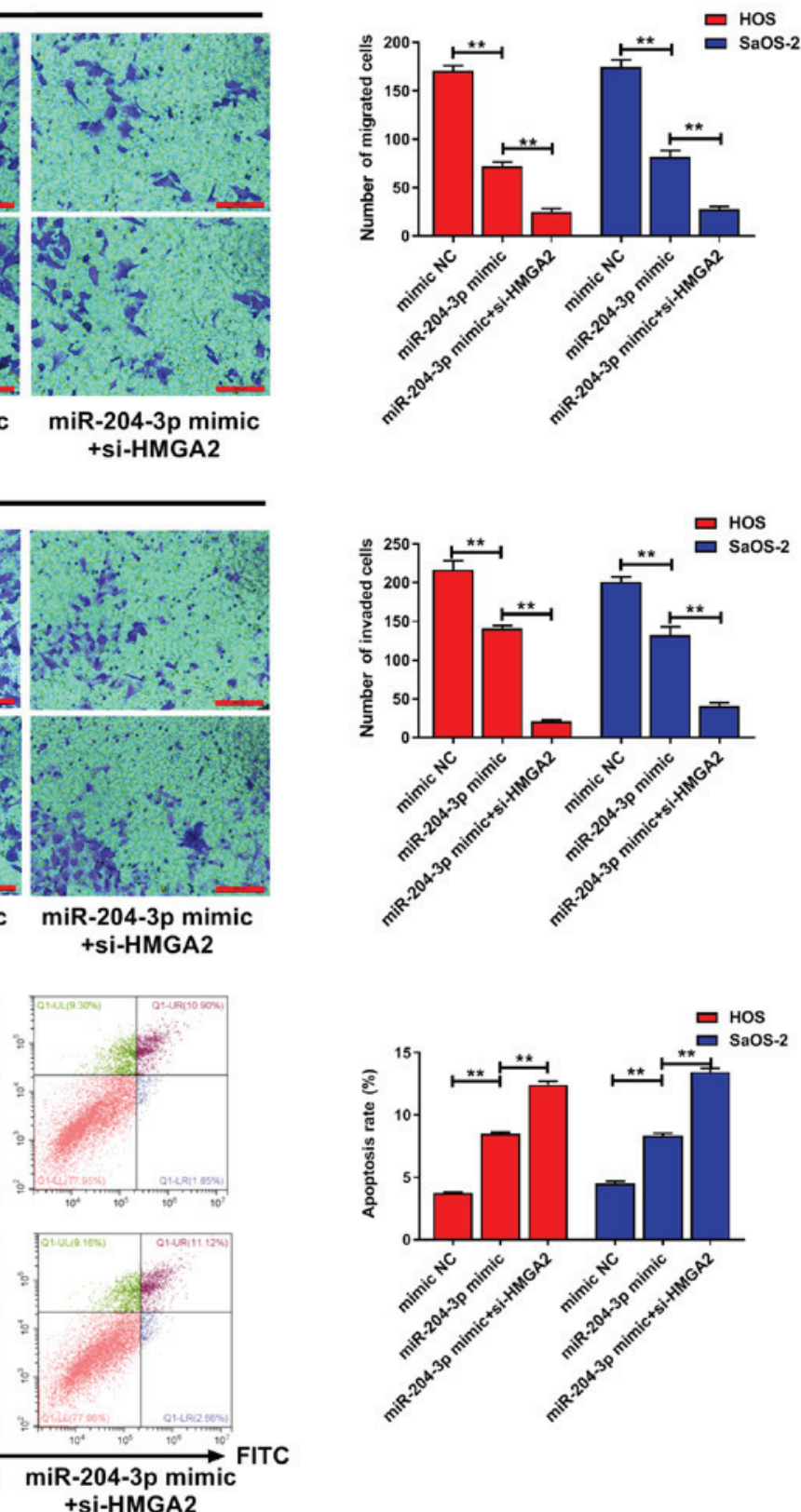

Figure 6. miR-204-3p inhibits OS cell proliferation, migration and invasion, and promotes OS cell apoptosis by targeting HMGA2. (A) Transfection efficiency of si-HMGA2. (B) Cell Counting Kit-8 assays were performed to assess the effect of miR-204-3p and HMGA2 on HOS and SaOS-2 cell proliferation. Transwell assays were performed to determine the effect of miR-204-3p and HMGA2 on HOS and SaOS-2 cell (C) migration and (D) invasion (scale bar, $500 \mu \mathrm{m}$ ). (E) Flow cytometry was performed to determine the effect of miR-204-3p and HMGA2 on HOS and SaOS-2 cell apoptosis. *P P $<0.01$ vs. si-NC. miR, microRNA; OS, osteosarcoma; si, small interfering RNA; HMGA2, high mobility group A2; NC, negative control; OD, optical density; d, days.

Furthermore, high expression levels of circUBAP2 in patients with OS were associated with lower survival rates compared with low expression levels of circUBAP2 in patients with OS, suggesting that circUBAP2 might serve as a prognosis 


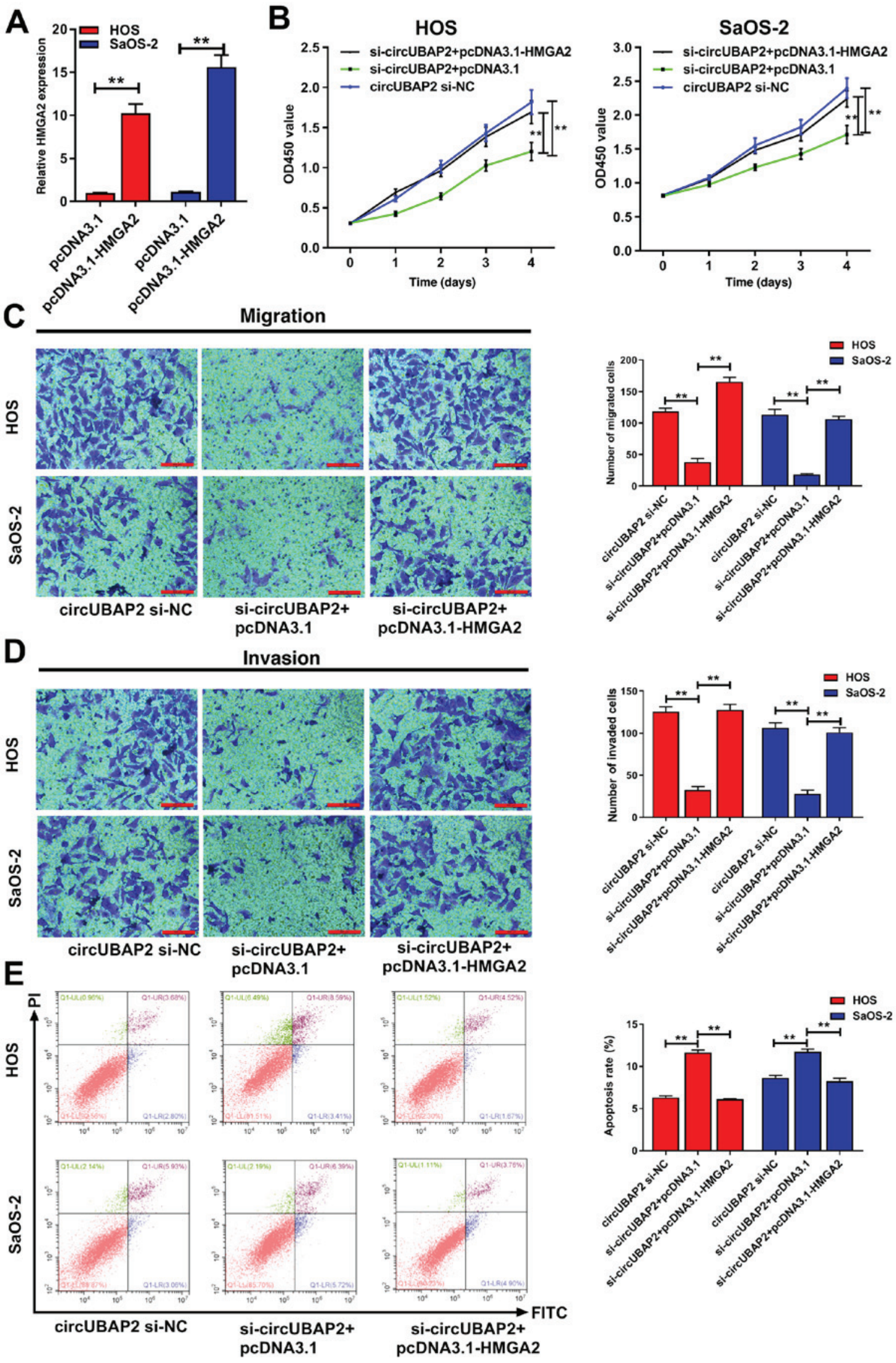

Figure 7. circUBAP2 promotes OS cell proliferation, migration and invasion, and inhibits OS cell apoptosis by regulating HMGA2. (A) Transfection efficiency of pcDNA3.1-HMGA2. (B) Effect of circUBAP2 and HMGA2 on HOS and SaOS-2 cell proliferation as determined by performing Cell Counting Kit-8 assays. (C) Effect of circUBAP2 and HMGA2 on HOS and SaOS-2 cell (C) migration and (D) invasion as determined by performing Transwell assays (scale bar, $500 \mu \mathrm{m}$ ). (E) Effect of circUBAP2 and HMGA2 on HOS and SaOS-2 cell apoptosis as determined via flow cytometry. ${ }^{* *} \mathrm{P}<0.01 \mathrm{vs}$. pcDNA3.1. circ, circular RNA; UBAP2, ubiquitin associated protein 2; OS, osteosarcoma; HMGA2, high mobility group A2; si, small interfering RNA; NC, negative control; OD, optical density; d, days. 
target for patients with OS. Moreover, circUBAP2 knockdown significantly inhibited OS cell proliferation, migration and invasion, and promoted OS cell apoptosis compared with the si-NC group.

Increasing evidence has also suggested that circRNAs perform regulatory roles as miRNA 'sponges' (40-42). To further investigate the mechanism underlying circUBAP2 in OS, the present study aimed to identify miRNAs that could bind to circUBAP2. Through bioinformatics analysis, miR-204-3p was identified as a target of circUBAP2. Subsequently, the dual-luciferase reporter and RIP assay results confirmed that circUBAP2 bound miR-204-3p, which is a known tumor suppressor in bladder cancer (27). Furthermore, miR-204-3p expression was significantly downregulated in OS tissues and cell lines compared with paracancerous tissues and hFOB1.19 cells, respectively. In addition, miR-204-3p expression was negatively correlated with circUBAP 2 expression in OS tissues. miR-204-3p overexpression significantly enhanced the inhibitory effect of circUBAP2 knockdown on OS cell proliferation, migration and invasion, whereas miR-204-3p overexpression significantly inhibited circUBAP2 knockdown-induced apoptosis.

As a transcription factor, HMGA2 can regulate the transcription and activation of a large number of genes, especially those associated with cell proliferation and apoptosis (58-61). The present study demonstrated that HMGA2 was a target of miR-204-3p. HMGA2 expression was significantly upregulated in OS tissues compared with paracancerous tissues. Furthermore, HMGA 2 knockdown significantly enhanced miR-204-3p overexpression-mediated effects on OS cell proliferation, migration, invasion and apoptosis. Based on the finding that miR-204-3p was associated with circUBAP2-mediated promotion of OS cell malignant behavior, it was hypothesized that circUBAP2 promoted OS cell malignant behavior by regulating HMGA2. Compared with the si-NC group, circUBAP2 knockdown significantly decreased cell proliferation, migration and invasion, and enhanced cell apoptosis, whereas these effects were significantly reversed by HMGA2 overexpression, which further supported our hypothesis. Therefore, the present study demonstrated that the novel circUBAP2/miR-204-3p/HMGA2 axis was involved in the OS malignant phenotype, suggesting that a circRNA-miRNA-mRNA regulatory network is involved in the tumorigenesis and development of OS.

However, the present study had a number of limitations. For example, although HMGA2 has been reported to be closely associated with the EMT process in tumors (33-35), the expression levels of EMT-associated proteins were not determined in the present study. Also, effects of HMGA2 alone on $\mathrm{OS}$ cell proliferation and migration require further investigation. Another potential limitation of present study is that only miR-204-3p mimic was used to investigate the effects of the circUBAP2/miR-204-3p/HMGA2 signaling pathway on OS and an miRNA-204-3p inhibitor was not used. Therefore, future studies should make use of an miR-204-3p inhibitor. In addition, the effect of circUBAP2 on tumor growth should be verified in vivo. Finally, only 42 OS tissues were used in the present study, which was a small number of samples, thus a larger number of tissue specimens should be utilized in future studies.
In conclusion, to the best of our knowledge, the present study demonstrated that circUBAP2 was highly expressed and served as an oncogene in OS for the first time. Moreover, the results of the present study indicated that the circUBAP2/miR-204-3p/HMGA2 signaling pathway might be involved in OS progression. Therefore, a novel regulatory network comprising circUBAP2/miR-204-3p/HMGA2 might serve as a potential therapeutic target for OS.

\section{Acknowledgements}

Not applicable.

\section{Funding}

No funding was received.

\section{Availability of data and materials}

The datasets used during the present study are available from the corresponding author upon reasonable request.

\section{Authors' contributions}

WM designed the study, performed the experiments and drafted the manuscript. NX and JZ analyzed the data. DW, $X Y$ and LL performed the experiments, analyzed the data and revised the manuscript. QX contributed to the conception and design of the study, analyzed the data and managed the project administration. WM and QX confirmed the authenticity of all the raw data. All authors read and approved the manuscript.

\section{Ethics approval and consent to participate}

The present study was approved by the Ethics Committee of Zhengzhou University (approval no. 201908). Written informed consent was obtained from all patients.

\section{Patient consent for publication}

Not applicable.

\section{Competing interests}

The authors declare that they have no competing interests.

\section{References}

1. Jaffe N: Osteosarcoma: Review of the past, impact on the future. The American experience. Cancer Treat Res 152: 239-262, 2009

2. Biazzo A and De Paolis M: Multidisciplinary approach to osteosarcoma. Acta Orthop Belg 82: 690-698, 2016.

3. Xiao X, Wang W and Wang Z: The role of chemotherapy for metastatic, relapsed and refractory osteosarcoma. Paediatr Drugs 16: 503-512, 2014.

4. Memczak S, Jens M, Elefsinioti A, Torti F, Krueger J, Rybak A, Maier L, Mackowiak SD, Gregersen LH, Munschauer M, et al: Circular RNAs are a large class of animal RNAs with regulatory potency. Nature 495: 333-338, 2013.

5. Rybak-Wolf A, Stottmeister C, Glažar P, Jens M, Pino N, Giusti S, Hanan M, Behm M, Bartok O, Ashwal-Fluss R, et al: Circular RNAs in the mammalian brain are highly abundant, conserved, and dynamically expressed. Mol Cell 58: 870-885, 2015. 
6. Wang G, Li Y, Liu Z, Ma X, Li M, Lu Q, Li Y, Lu Z, Niu L, Fan Z, et al: Circular RNA circ_0124644 exacerbates the ox-LDL-induced endothelial injury in human vascular endothelial cells through regulating PAPP-A by acting as a sponge of miR-149-5p. Mol Cell Biochem 471: 51-61, 2020.

7. Li J,Li P,Zhang G, Qin P,Zhang D and Zhao W: CircRNA TADA2A relieves idiopathic pulmonary fibrosis by inhibiting proliferation and activation of fibroblasts. Cell Death Dis 11: 553,2020.

8. Guo X, Dai X, Liu J, Cheng A, Qin C and Wang Z: Circular RNA circREPS2 acts as a sponge of miR-558 to suppress gastric cancer progression by regulating RUNX3/ $\beta$-catenin signaling. Mol Ther Nucleic Acids 21: 577-591, 2020.

9. Si X, Zheng H, Wei G, Li M, Li W, Wang H, Guo H, Sun J, Li C, Zhong S, et al: circRNA Hipk3 induces cardiac regeneration after myocardial infarction in mice by binding to Notch 1 and miR-133a. Mol Ther Nucleic Acids 21: 636-655, 2020.

10. Li Y, Lv Z, Zhang J, Ma Q, Li Q, Song L, Gong L, Zhu Y, Li X, Hao Y, et al: Profiling of differentially expressed circular RNAs in peripheral blood mononuclear cells from Alzheimer's disease patients. Metab Brain Dis 35: 201-213, 2020.

11. Schulte C, Barwari T, Joshi A, Theofilatos K, Zampetaki A, Barallobre-Barreiro J, Singh B, Sörensen NA, Neumann JT, Zeller T, et al: Comparative analysis of circulating noncoding RNAs versus protein biomarkers in the detection of myocardial injury. Circ Res 125: 328-340, 2019

12. Sun H, Xi P, Sun Z, Wang Q, Zhu B, Zhou J, Jin H, Zheng W, Tang W, Cao H, et al: Circ-SFMBT2 promotes the proliferation of gastric cancer cells through sponging miR-182-5p to enhance CREB1 expression. Cancer Manag Res 10: 5725-5734, 2018.

13. Yuan P,Lei L, Dong S and Liu D: CircularRNA hsa_circ_0068033 acts as a diagnostic biomarker and suppresses the progression of breast cancer through sponging miR-659. OncoTargets Ther 13 1921-1929, 2020.

14. Ren T, Liu C, Hou J and Shan F: Hsa_circ_0043265 suppresses proliferation, metastasis, EMT and promotes apoptosis in non-small cell lung cancer through miR-25-3p/FOXP2 pathway. OncoTargets Ther 13: 3867-3880, 2020.

15. Quan J, Dong D, Lun Y, Sun B, Sun H, Wang Q and Yuan G: Circular RNA circHIAT1 inhibits proliferation and epithelial-mesenchymal transition of gastric cancer cell lines through downregulation of miR-21. J Biochem Mol Toxicol 34 e22458, 2020.

16. Wang J, Li H and Liang Z: circ-MYBL2 serves as a sponge for miR-361-3p promoting cervical cancer cells proliferation and invasion. OncoTargets Ther 12: 9957-9964, 2019.

17. Sun J, Yin A, Zhang W, Lv J, Liang Y, Li H, Li Y and Li X: CircUBAP2 inhibits proliferation and metastasis of clear cell renal cell carcinoma via targeting miR-148a-3p/FOXK2 pathway. Cell Transplant: May 19, 2020 (Epub ahead of print). doi: $10.1177 / 0963689720925751$

18. Wu Y, Zhi L, Zhao Y, Yang L and Cai F: Knockdown of circular RNA UBAP2 inhibits the malignant behaviours of esophagea squamous cell carcinoma by microRNA-422a/Rab10 axis. Clin Exp Pharmacol Physiol 47: 1283-1290, 2020.

19. Wang S, Li Q, Wang Y, Li X, Wang R, Kang Y, Xue X, Meng R, Wei Q and Feng X: Upregulation of circ-UBAP2 predicts poor prognosis and promotes triple-negative breast cancer progression through the miR-661/MTA1 pathway. Biochem Biophys Res Commun 505: 996-1002, 2018

20. Sheng M, Wei N, Yang HY, Yan M, Zhao QX and Jing LJ: CircRNA UBAP2 promotes the progression of ovarian cancer by sponging microRNA-144. Eur Rev Med Pharmacol Sci 23 7283-7294, 2019.

21. Zhang H, Wang G, Ding C, Liu P, Wang R, Ding W, Tong D, Wu D, Li C, Wei Q, et al: Increased circular RNA UBAP2 acts as a sponge of miR-143 to promote osteosarcoma progression. Oncotarget 8: 61687-61697, 2017.

22. Wu H, Li W, Zhu S, Zhang D and Zhang M: Circular RNA circUBAP2 regulates proliferation and invasion of osteosarcoma cells through miR-641/YAP1 axis. Cancer Cell Int 20: 223, 2020.

23. Bartel DP: MicroRNAs: Target recognition and regulatory functions. Cell 136: 215-233, 2009

24. Wang Y, Zhang S, Xu Y, Zhang Y, Guan H, Li X, Li Y and Wang Y: Upregulation of miR-192 inhibits cell growth and invasion and induces cell apoptosis by targeting TCF7 in human osteosarcoma. Tumour Biol 37: 15211-15220, 2016

25. Sun X, Xu Y, Zhang S, Li X, Wang Y, Zhang Y, Zhao X, Li Y and Wang Y: MicroRNA-183 suppresses the vitality, invasion and migration of human osteosarcoma cells by targeting metastasis-associated protein 1. Exp Ther Med 15: 5058-5064, 2018.
26. Gao S, Wang K and Wang X: miR-375 targeting autophagyrelated 2B (ATG2B) suppresses autophagy and tumorigenesis in cisplatin-resistant osteosarcoma cells. Neoplasma 67: 724-734, 2020.

27. Guo J, Zhao P, Liu Z, Li Z, Yuan Y, Zhang X, Yu Z, Fang J and Xiao K: MiR-204-3p inhibited the proliferation of bladder cancer cells via modulating lactate dehydrogenase-mediated glycolysis. Front Oncol 9: 1242, 2019.

28. Chen PH, Chang CK, Shih CM, Cheng $\mathrm{CH}$, Lin CW, Lee CC, Liu AJ, Ho KH and Chen KC: The miR-204-3p-targeted IGFBP2 pathway is involved in xanthohumol-induced glioma cell apoptotic death. Neuropharmacology 110: 362-375, 2016.

29. Mansoori B, Duijf PHG, Mohammadi A, Najafi S, Roshani E, Shanehbandi D, Hajiasgharzadeh K, Shirjang S, Ditzel HJ, Kazemi T, et al: Overexpression of HMGA2 in breast cancer promotes cell proliferation, migration, invasion and stemness. Expert Opin Ther Targets 24: 1-11, 2020.

30. Guo X, Shi J, Wen Y, Li M, Li Q, Li X and Li J: Increased high-mobility group A2 correlates with lymph node metastasis and prognosis of non-small cell lung cancer. Cancer Biomark 21: 547-555, 2018

31. Guo HH, Wang YZ, Zhang ZK, Li MZ, Tian XD and Yang YM: High mobility group AT-hook 2 promotes tumorigenicity of pancreatic cancer cells via upregulating ANLN. Exp Cell Res 393: 112088, 2020.

32. Venkatesan N, Kandalam M, Pasricha G, Sumantran V, Manfioletti G, Ono SJ, Reddy MA and Krishnakumar S: Expression of high mobility group A2 protein in retinoblastoma and its association with clinicopathologic features. J Pediatr Hematol Oncol 31: 209-214, 2009.

33. Zha L, Zhang J, Tang W, Zhang N, He M, Guo Y and Wang Z HMGA2 elicits EMT by activating the Wnt/ $\beta$-catenin pathway in gastric cancer. Dig Dis Sci 58: 724-733, 2013.

34. Zhao XP, Zhang H, Jiao JY, Tang DX, Wu YL and Pan CB: Overexpression of HMGA2 promotes tongue cancer metastasis through EMT pathway. J Transl Med 14: 26, 2016.

35. Hawsawi O, Henderson V, Burton LJ, Dougan J, Nagappan P and Odero-Marah V: High mobility group A2 (HMGA2) promotes EMT via MAPK pathway in prostate cancer. Biochem Biophys Res Commun 504: 196-202, 2018.

36. Wang YD, Mao JD, Wang JF and Xu MQ: MiR-590 suppresses proliferationandinducesapoptosisin pancreaticcancerbytargeting high mobility group A2. Technol Cancer Res Treat: Jun 26, 2020 (Epub ahead of print). doi: 10.1177/1533033820928143

37. Huang WT,Zhang H, Jin Z, Li K, Hu C, Li ML and Situ J: MiR-219-5p inhibits prostate cancer cell growth and metastasis by targeting HMGA2. Eur Rev Med Pharmacol Sci 24: 4710-4718, 2020.

38. Zhou ZG, Xu C, Dong Z, Wang YP, Duan JY and Yan CQ: MiR-497 inhibits cell proliferation and invasion ability by targeting HMGA2 in pancreatic ductal adenocarcinoma. Eur Rev Med Pharmacol Sci 24: 122-129, 2020.

39. Jiao D, Liu Y and Tian Z: microRNA-493 inhibits tongue squamous cell carcinoma oncogenicity via directly targeting HMGA2. OncoTargets Ther 12: 6947-6959, 2019.

40. Sang Y, Chen B, Song X, Li Y, Liang Y, Han D, Zhang N, Zhang H, Liu Y, Chen T, et al: circRNA_0025202 Regulates Tamoxifen Sensitivity and Tumor Progression via Regulating the miR-182-5p/ FOXO3a Axis in Breast Cancer. Mol Ther 27: 1638-1652, 2019.

41. Song T, Xu A, Zhang Z, Gao F, Zhao L, Chen X, Gao J and Kong X CircRNA hsa_circRNA_101996 increases cervical cancer proliferation and invasion through activating TPX2 expression by restraining miR-8075. J Cell Physiol 234: 14296-14305, 2019.

42. Xu JZ, Shao CC, Wang XJ, Zhao X, Chen JQ, Ouyang YX, Feng J, Zhang F, Huang WH, Ying Q, et al: circTADA2As suppress breast cancer progression and metastasis via targeting miR-203a-3p/SOCS3 axis. Cell Death Dis 10: 175, 2019.

43. Livak KJ and Schmittgen TD: Analysis of relative gene expression data using real-time quantitative PCR and the 2(-Delta Delta $\mathrm{C}(\mathrm{T})$ ) method. Methods 25: 402-408, 2001.

44. Cates JM: Comparison of the AJCC, MSTS, and modified spanier systems for clinical and pathologic staging of osteosarcoma. Am J Surg Pathol 41: 405-413, 2017.

45. He H, Zhao X, Zhu Z, Du L, Chen E, Liu S, Li Q, Dong J, Yang J and Lei L: MicroRNA-3191 promotes migration and invasion by downregulating TGFBR2 in colorectal cancer. J Biochem Mol Toxicol 33: e22308, 2019.

46. Wang Y, Cheng N and Luo J: Downregulation of lncRNA ANRIL represses tumorigenicity and enhances cisplatin-induced cytotoxicity via regulating microRNA let-7a in nasopharyngeal carcinoma. J Biochem Mol Toxicol 31: e21904, 2017. 
47. Li Z, Li Y, Li Y, Ren K, Li X, Han X and Wang J: Long non-coding RNA H19 promotes the proliferation and invasion of breast cancer through upregulating DNMT1 expression by sponging miR-152. J Biochem Mol Toxicol 31: e21933, 2017.

48. Guan H, Mei Y, Mi Y, Li C, Sun X, Zhao X, Liu J, Cao W, Li Y and Wang Y: Downregulation of lncRNA ANRIL suppresses growth and metastasis in human osteosarcoma cells. OncoTargets Ther 11: 4893-4899, 2018.

49. Guan H, Liu J, Lv P, Zhou L, Zhang J and Cao W: MicroRNA 590 inhibits migration, invasion and epithelial to mesenchymal transition of esophageal squamous cell carcinoma by targeting low density lipoprotein receptor related protein 6. Oncol Rep 44: 1385-1392, 2020.

50. Guan H, Shang G, Cui Y, Liu J, Sun X, Cao W, Wang Y and Li Y: Long noncoding RNA APTR contributes to osteosarcoma progression through repression of miR-132-3p and upregulation of yes-associated protein 1. J Cell Physiol 234: 8998-9007, 2019.

51. Gao PF, Huang D, Wen JY, Liu W and Zhang HW: Advances in the role of exosomal non-coding RNA in the development diagnosis, and treatment of gastric cancer (Review). Mol Clin Oncol 13: 101-108, 2020.

52. Zhang C, Huo ST, Wu Z, Chen L, Wen C, Chen H, Du WW, Wu N, Guan D, Lian S, et al: Rapid development of targeting circRNAs in cardiovascular diseases. Mol Ther Nucleic Acids 21: 568-576, 2020.

53. Ma Y, Liu Y and Jiang Z: CircRNAs: A new perspective of biomarkers in the nervous system. Biomed Pharmacother 128: $110251,2020$.

54. Filardi T, Catanzaro G, Mardente S, Zicari A, Santangelo C, Lenzi A, Morano S and Ferretti E: Non-coding RNA: Role in gestational diabetes pathophysiology and complications. Int J Mol Sci 21: 4020, 2020.
55. Lodde V, Murgia G, Simula ER, Steri M, Floris M and Idda ML: Long noncoding RNAs and circular RNAs in autoimmune diseases. Biomolecules 10: E1044, 2020.

56. Hao Y, Xi J, Peng Y, Bian B, Hao G, Xi Y and Zhang Z: Circular RNA Circ_0016760 modulates non-small-cell lung cancer growth through the miR-577/ZBTB7A axis. Cancer Manag Res 12: 5561-5574, 2020.

57. Wang K, Gan TY, Li N, Liu CY, Zhou LY, Gao JN, Chen C, Yan KW, Ponnusamy M, Zhang YH, et al: Circular RNA mediates cardiomyocyte death via miRNA-dependent upregulation of MTP18 expression. Cell Death Differ 24: 1111-1120, 2017.

58. Tan L, Wei X, Zheng L, Zeng J, Liu H, Yang S and Tan H: Amplified HMGA2 promotes cell growth by regulating Akt pathway in AML. J Cancer Res Clin Oncol 142: 389-399, 2016.

59. Ikeda $K$, Ogawa $K$ and Takeishi $Y$ : The role of HMGA2 in the proliferation and expansion of a hematopoietic cell in myeloproliferative neoplasms. Fukushima J Med Sci 58: 91-100, 2012.

60. Pallante P, Sepe R, Puca F and Fusco A: High mobility group a proteins as tumor markers. Front Med (Lausanne) 2: 15, 2015.

61. Mansoori B, Mohammadi A, Shirjang S and Baradaran B: HMGI-C suppressing induces P53/caspase9 axis to regulate apoptosis in breast adenocarcinoma cells. Cell Cycle 15: 2585-2592, 2016.

c) (i) $\Theta$ This work is licensed under a Creative Commons International (CC BY-NC-ND 4.0) License. 